\title{
Long-term Prediction of Bridge Element Performance Using Time Delay Neural Networks (TDNNs)
}

\author{
Jaeho Lee \\ Research fellow, \\ Griffith School of Engineering, \\ Griffith University, Australia \\ j.lee@griffith.edu.au
}

\author{
Hong Guan \\ Associate Professor, \\ Griffith School of Engineering, \\ Griffith University, Australia \\ h.guan@griffith.edu.au
}

\author{
Michael Blumenstein \\ Associate Professor, \\ Executive, Dean (Research) SEET, \\ Griffith University, Australia \\ m.blumenstein@griffith.edu.au
}

\author{
Yew-Chaye Loo \\ Professor, \\ Director of International and \\ Professional Liaison for SEET Group \\ and Foundation Professor of Griffith \\ School of Engineering, Griffith \\ University, Australia \\ y.loo@griffith.edu.au
}

\section{Summary}

A bridge is principally designed to have a long service life. However, due to number factors, it could fail prematurely, and could cause loss of human life. In order to ensure the optimum bridge serviceability, systematic asset management is essential for effective decision-making of maintenance, repair and rehabilitation (MR\&R). Systematic asset management can be achieved by a computer-based bridge management system (BMS). Successful BMS development requires a reliable bridge deterioration model, which is the most crucial component in a BMS. Historical condition ratings obtained from biennial bridge inspections are a major resource for predicting future bridge deterioration via BMSs. However, available historical condition ratings from most bridge agencies are very limited, thus posing a major barrier for predicting reliable future bridge performance.

This paper presents the progressive research on the development of a reliable bridge deterioration model using advanced Artificial Intelligence (AI) techniques. The development is organised in three major steps: (1) generating unavailable past bridge element condition ratings using the Backward Prediction Model (BPM) - this helps to provide sufficient historical deterioration patterns for each element; (2) predicting long-term condition ratings based on the outcome of Step 1 using Time Delay Neural Networks (TDNNs); and (3) improving long-term prediction accuracy of Step 2 by employing Case-based Reasoning (CBR). This paper mainly focuses on the first two steps of the research. Promising results are reported for the reliable long-term prediction of bridge element performance.

Keywords: Maintenance, Repair and Rehabilitation (MR\&R); Bridge Management System (BMS); Artificial Intelligence (AI); Backward Prediction Model (BPM), Time Delay Neural Networks (TDNNs).

\section{Introduction}

The long-term bridge needs can be determined by future bridge condition ratings using the deterioration model. The results from the deterioration model can be also used as major input resources in other BMS analysis models, such as cost analysis and maintenance prioritisation, in BMSs for establishing a long-term MR\&R strategy. Consequently, without a reliable deterioration model, the outcome of other BMS analysis modules can also become unreliable.

To obtain reliable outcomes from a deterioration model, sufficient amount of condition rating records are required for long-term predictions. As such, keeping up-to-date such data is crucial. Although most bridge agencies in the past have conducted inspections and maintenances, past 
bridge inspection records, being non-quantitative, are incompatible with what is required by typical BMSs. Such incompatibility is the major cause of deficiency of historical structural information for BMSs. Because of this sheer lack of usable inspection records, current deterioration modelling techniques have been designed to use only a few sets of recent structural condition ratings, thus resulting in unreliable predictions of future structural condition ratings in any computational prediction methods. The amount of required datasets is usually much larger than the target prediction datasets in order to obtain reliable prediction results.

In relation to condition rating availability and deterioration modelling techniques, there are a number of shortcomings related to the use of BMSs from the perspective of bridge agencies. Inter alia these are [1-6]: (1) Commercial BMS software has been used for less than two decades and even those bridge agencies which implemented BMSs from an early stage, would have usually approximately 4 to 8 biennial inspection records at their disposal; (2) Bridge condition ratings normally do not change much over short time periods; (3) Approximately $60 \%$ of BMS analytical processes rely heavily on periodic bridge inspection results; (4) Interactive deterioration mechanism effects between or among structure elements are ignored; (5) One of the assumptions made in most deterioration models is that future conditions are dependent only on the current structural conditions; (6) The predicted bridge condition ratings do not acceptably match the real situation; (7) Bridge condition rating variances in the small number of historical datasets inevitably lead to unreliable structural performance predictions by current deterioration models in BMSs.

A recent study has been focused mainly on the problem of insufficient historical condition rating records to overcome the unrealistic outcome of current deterioration modelling. This study has been presented the method for generating unavailable historical bridge condition ratings [4]. This method referred to as the Artificial Intelligence (AI) based Backward Prediction Model (BPM), which provides unknown historical bridge deterioration patterns to assist in predicting reliable long-term bridge deteriorations. A feasibility study is conducted for the bridge deterioration modelling using BPM-generated historical condition ratings for individual bridge elements. This is achieved by employing neural network techniques, i.e. Backpropagation algorithm and Time-Delay Neural Networks (TDNNs). The outcome of the present study would be useful to provide alternative ways for developing long-term deterioration model using very limited bridge condition ratings.

\section{Outline of the study}

The concept of the present study, shown in Fig. 1, illustrates that the abovementioned limitations of the current deterioration modelling can be minimised. This study has two major components: (1) generating unavailable historical condition ratings (years from t0 to t1) by using the BPM methodology. It is to establish a comprehensive bridge condition ratings (years from t0 to t2) which contains more historical deterioration patterns than the shear amount of available condition ratings (from $\mathrm{t} 1$ to $\mathrm{t} 2$ ); (2) the outcomes from (1) are to predict long-term performance of individual bridge elements using time-series neural network technique.

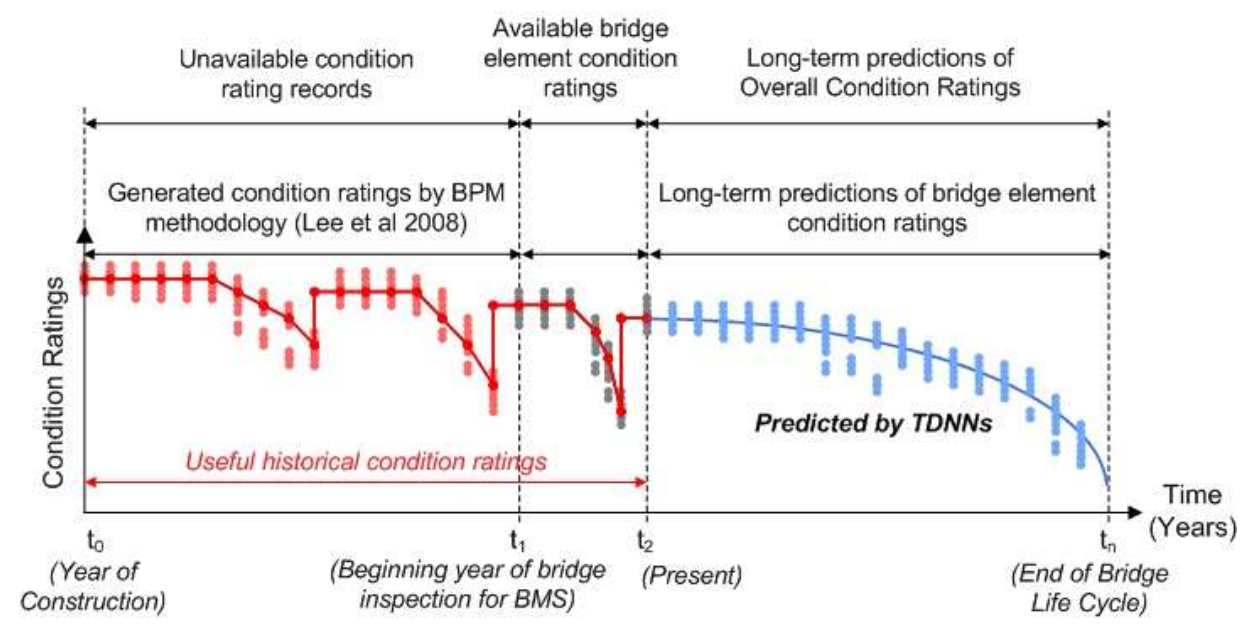

Fig. 1: The proposed deterioration model 


\section{Feasibility study}

A feasibility study for the present study is hereby conducted by using the BPM methodology in conjunction with the Time-Delay Neural Networks (TDNNs) technique. The proposed deterioration model has two-stage procedure: Stage 1 - generating historical condition rating by using BPM methodology; Stage 2- Predicting long-term performance of bridge elements by using TDNN technique.

Fig. 2 describes the timeframe of the proposed deterioration model. Only five sets of actual condition ratings (years from 1996 to 2004 with a 2-year increment) are available for both BPM and TDNN for long-term predictions. The timeframe shows, for different years: (a) available condition ratings; (b) BPM inputs; (c) BPM results viz the generated historical condition ratings; (d) input for validation; (e) results for BPM validation; (f) TDNN input; (g) TDNN prediction results; and (f) cross-validation of TDNN results.

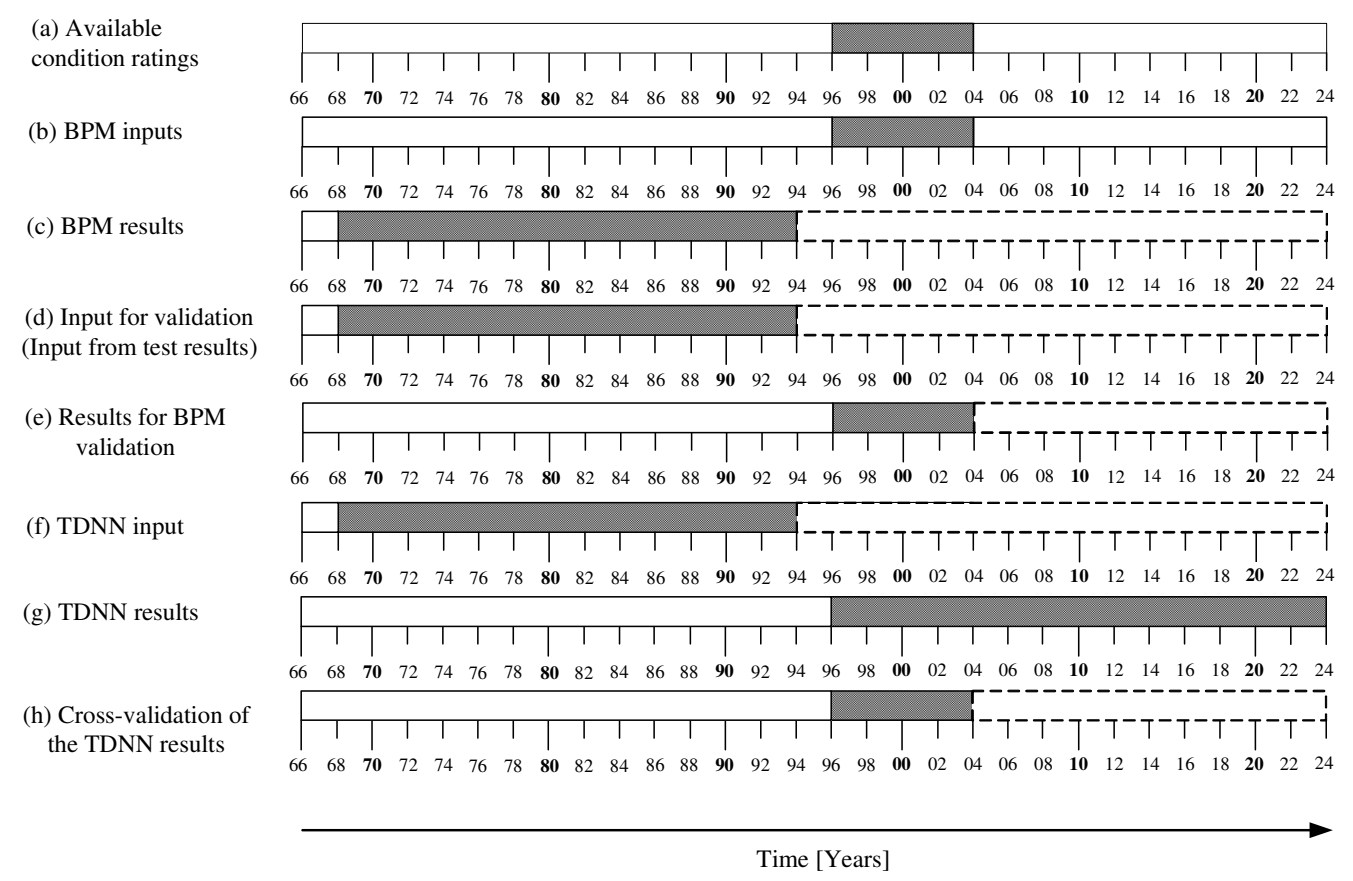

Fig. 2: Timeframe of the proposed deterioration model (Element \#234 in Bridge \#0301xxxx1)

Table 1: Raw data of actual condition ratings (Element \#234 on Bridge \#0301xxxx1)

\begin{tabular}{ccccccc}
\hline $\begin{array}{c}\text { Year of } \\
\text { inspection }\end{array}$ & $\begin{array}{c}\text { Total } \\
\text { Quantity } \\
(\%)\end{array}$ & $\begin{array}{c}\text { CS1 } \\
(\%)\end{array}$ & $\begin{array}{c}\text { CS2 } \\
(\%)\end{array}$ & $\begin{array}{c}\text { CS3 } \\
(\%)\end{array}$ & $\begin{array}{c}\text { CS4 } \\
(\%)\end{array}$ & $\begin{array}{c}\text { CS5 } \\
(\%)\end{array}$ \\
\hline 1996 & 100 & 80 & 14 & 6 & 0 & 0 \\
1998 & 100 & 80 & 14 & 6 & 0 & 0 \\
2000 & 100 & 80 & 14 & 6 & 0 & 0 \\
2002 & 100 & 80 & 19 & 1 & 0 & 0 \\
2004 & 100 & 80 & 19 & 1 & 0 & 0 \\
\hline Average $(\%)$ & 100 & 80.0 & 16.2 & 3.8 & 0 & 0 \\
\hline
\end{tabular}

In this feasibility study uses sample dataset (see Table 1) provided by the Maryland Department of Transport (DoT), USA. It is one superstructure element - Reinforced Concrete Pier Cap. The Condition Index (CI) of BMS condition ratings is scaled between CS1 (excellent) and 5 (fail) for the bridge element

\subsection{Generating historical condition ratings}

The BPM methodology [4] is used to generate historical condition ratings. The actual element-level bridge inspection records (years from 1996 to 2004) are correlated with relevant non-bridge factors, 
such as traffic volume and climatic condition, in the neural network training session to generate missing historical condition ratings (years from 1968 to 1994) in the testing session. The generated condition ratings for each year contain 66 cases which are the combined number of learning rates (lr: 0.0-0.5) and momentum coefficients (mc: 0.0-1.0) in the neural network configurations. The number 66 also represents the total quantity of a given bridge element. The forward comparison method is used in the BPM methodology to validate the generated condition ratings (years from 1996 to 2004). It is comparison between the generated condition ratings and the actual BMS condition ratings.

The average quantity of each CS on Element \#234 between 1996 and 2004 is about 80\%, 16.2\% and $3.8 \%$ of the total element quantities in CS1, CS2 and CS3 respectively. The BPM generates historical condition ratings from 1968 to 1994 in three different proportions of the element quantity as shown in Fig. 3.

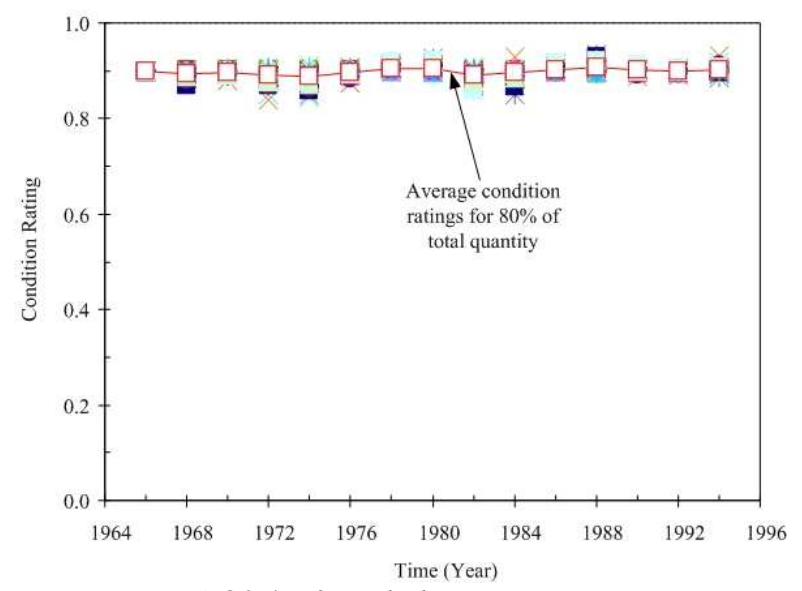

(a) $80 \%$ of total element quantity

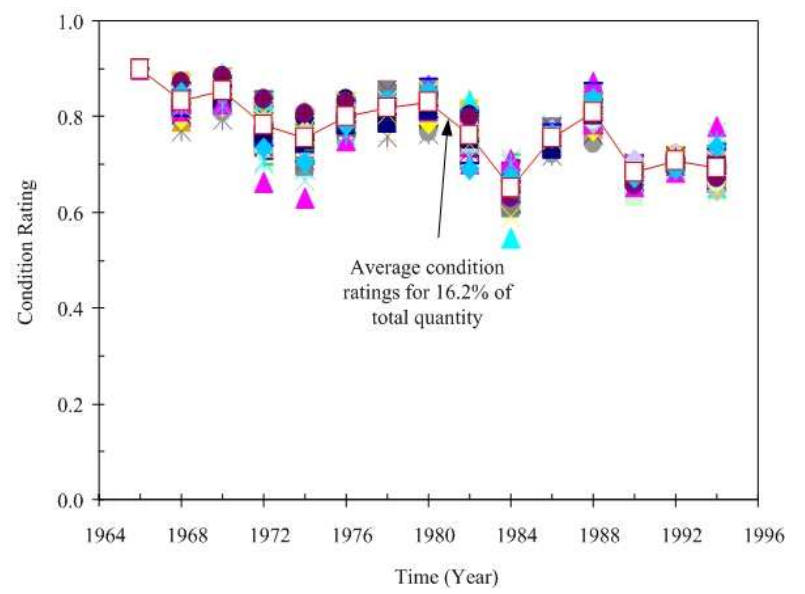

(b) $16.2 \%$ of total element quantity

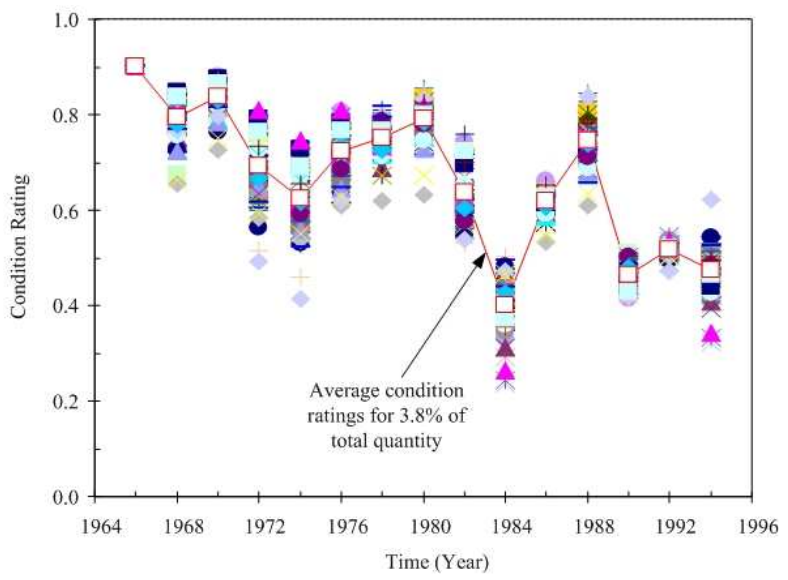

(c) $3.8 \%$ of total element quantity

Fig. 3 BPM results (Element \#234, Bridge \#0301xxxx1)

To validate the results of the BPM, generated historical condition ratings (1968-1994) are used as input datasets in this test to generate the condition ratings for the present years (1996-2004). The BPM-generated condition ratings are then directly compared with the existing condition rating datasets. Table 2 shows the final results from the BPM and their prediction errors. For all five CSs, the yearly average prediction differences are less than $\pm 10 \%$ which is acceptable. This suggests that, the generated historical condition ratings (1966-1994) by the BPM can be used with confidence as input data for predicting long-term performance of bridge elements in Stage II. 
Table 2: $\quad$ Prediction errors of the BPM using forward comparisons (Bridge \#0301xxxx1)

\begin{tabular}{|c|c|c|c|c|c|c|c|}
\hline \multicolumn{2}{|c|}{ Year } & $\begin{array}{l}\text { CS1 } \\
(\%)\end{array}$ & $\begin{array}{l}\mathrm{CS} 2 \\
(\%)\end{array}$ & $\begin{array}{l}\text { CS3 } \\
(\%)\end{array}$ & $\begin{array}{l}\text { CS4 } \\
(\%)\end{array}$ & $\begin{array}{l}\text { CS5 } \\
(\%)\end{array}$ & $\begin{array}{c}\text { Total } \\
(\%)\end{array}$ \\
\hline \multirow{4}{*}{1996} & $\mathrm{~A}$ & 87.75 & 10.98 & 1.15 & 0.11 & 0.02 & 100 \\
\hline & B & 80.00 & 14.29 & 5.71 & 0.00 & 0.00 & 100 \\
\hline & $\mathrm{C}$ & 7.75 & 3.31 & 4.56 & 0.11 & 0.02 & - \\
\hline & $\mathrm{D}$ & & & 3.15 & & & - \\
\hline \multirow{4}{*}{1998} & $\mathrm{~A}$ & 87.75 & 10.96 & 1.29 & 0.00 & 0.01 & 100 \\
\hline & B & 80.00 & 14.29 & 5.71 & 0.00 & 0.00 & 100 \\
\hline & $\mathrm{C}$ & 7.75 & 3.33 & 4.42 & 0.00 & 0.01 & - \\
\hline & $\mathrm{D}$ & & & 3.10 & & & - \\
\hline \multirow{4}{*}{2000} & $\mathrm{~A}$ & 90.57 & 8.53 & 0.87 & 0.03 & 0.01 & 100 \\
\hline & B & 80.00 & 14.29 & 5.71 & 0.00 & 0.00 & 100 \\
\hline & $\mathrm{C}$ & 10.57 & 5.76 & 4.84 & 0.03 & 0.01 & - \\
\hline & $\mathrm{D}$ & & & 4.24 & & & - \\
\hline \multirow{4}{*}{2002} & $\mathrm{~A}$ & 90.91 & 7.80 & 1.09 & 0.20 & 0.01 & 100 \\
\hline & B & 80.00 & 19.14 & 0.86 & 0.00 & 0.00 & 100 \\
\hline & $\mathrm{C}$ & 10.91 & 11.34 & 0.23 & 0.20 & 0.01 & - \\
\hline & $\mathrm{D}$ & & & 4.54 & & & - \\
\hline \multirow{4}{*}{2004} & $\mathrm{~A}$ & 87.75 & 11.20 & 1.03 & 0.02 & 0.01 & 100 \\
\hline & B & 80.00 & 19.14 & 0.86 & 0.00 & 0.00 & 100 \\
\hline & $\mathrm{C}$ & 7.75 & 7.94 & 0.17 & 0.02 & 0.01 & - \\
\hline & $\mathrm{D}$ & & & 3.18 & & & - \\
\hline
\end{tabular}

A: Results, B: Existing data, C: Difference, D: Average Difference

\subsection{Predicting long-term performance of bridge elements}

TDNNs have been acknowledged as the Neural Network Finite Impulse Response (NNFIR) and has been extensively used in numerous practical applications to solve non-linearity problems [7]. For the proposed TDNN model, a sigmoid transfer function is also selected as used in the BPM methodology due to nonlinear characteristic of bridge deterioration behaviour.

\section{In Stage 2, the BPM results} (years from 1968 to 1994) obtained from Stage 1 are used as TDNN inputs to estimate long-term bridge element performances. It is noted that the present study in this feasibility study is only considered "Do-nothing" - no maintenance effects in longterm prediction.

The generated historical condition ratings (years from 1968 to 1994) from the BPM methodology are used as TDNN inputs. These input data is converted to time sequences for time series prediction in the TDNN. The proposed TDNN provides only one-step ahead prediction at a time. The result of the first one-step-ahead prediction (i.e. year 1996) is added on to the original TDNN input (years from 1968 to 1994). Iterations of the above-described process are required for a given bridge element until the entire bridge elements' condition rating reaches zero. The number of yearly prediction by TDNN is also 66, which is in an identical form as the BPM outcomes obtained in Stage 1. Fig. 4 shows the results of long-term prediction of condition ratings (years from 1996 to 2070).

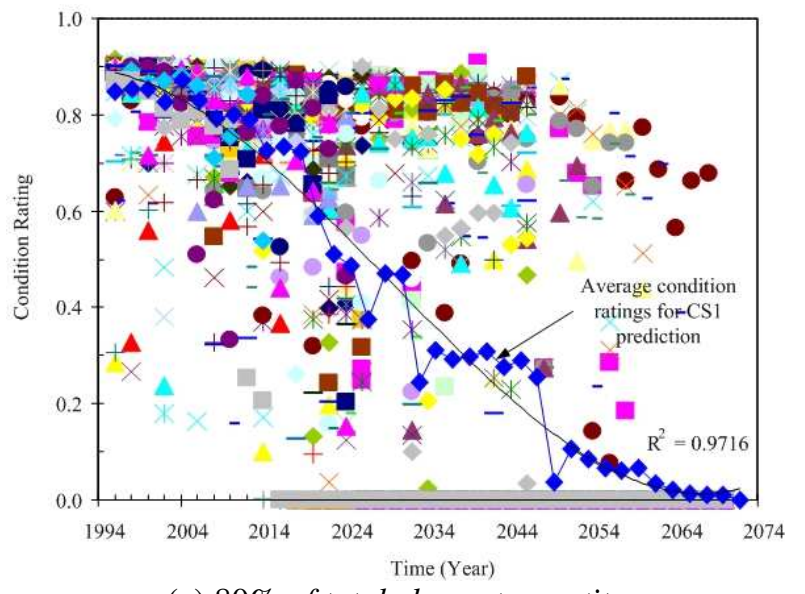

(a) $80 \%$ of total element quantity

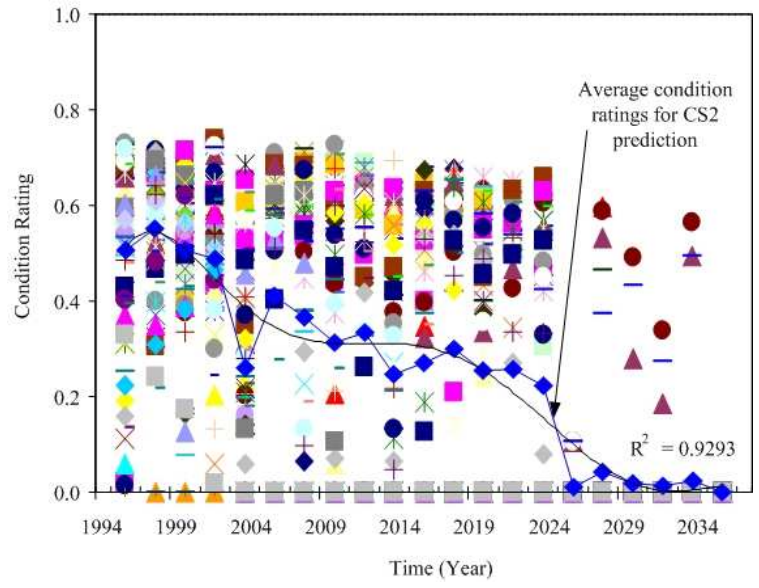

(b) $16.2 \%$ of total element quantity 


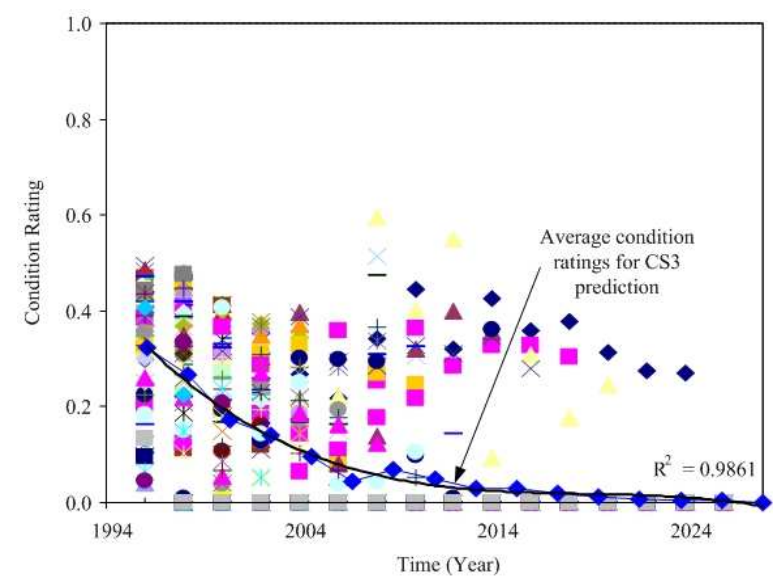

(c) $3.8 \%$ of total element quantity

Fig 4: Long-term prediction of condition rating - "Do Nothing" maintenance effect (Element \#234, Bridge \#0301 xxxx1)

Table 3: Cross-validation results (Bridge \#0301xxxx1)

In order to validate the outcome

\begin{tabular}{|c|c|c|c|c|c|c|c|c|}
\hline Year & & CS1 & CS2 & CS3 & CS4 & CS5 & Total & ction results and \\
\hline \multirow{4}{*}{1996} & $\mathrm{~A}(\%)$ & 75.78 & 12.29 & 5.75 & 4.66 & 1.53 & 100 & \multirow{4}{*}{$\begin{array}{l}\text { rating records for the same } \\
\text { years }(1996-2004) \text { are } \\
\text { compared. For this 5-year } \\
\text { period and } 5 \text { CSs, a total of } 25 \\
\text { cross-validation comparisons }\end{array}$} \\
\hline & $\mathrm{B}(\%)$ & 80.00 & 14.29 & 5.71 & 0.00 & 0.00 & & \\
\hline & $\mathrm{C}(\%)$ & 4.22 & 2.00 & 0.04 & 4.66 & 1.53 & & \\
\hline & $\mathrm{D}(\%)$ & & & 2.49 & & & - & \\
\hline \multirow{4}{*}{1998} & $\mathrm{~A}(\%)$ & 74.46 & 16.78 & 5.07 & 1.67 & 2.04 & \multirow{4}{*}{$\begin{array}{c}100 \\
100 \\
- \\
-\end{array}$} & \multirow{8}{*}{$\begin{array}{l}\text { are resulted and presented in } \\
\text { Table } 3 \text {. It is evident that the } \\
\text { yearly average prediction } \\
\text { differences are less than } \pm 10 \% \text {, } \\
\text { (i.e. year } 1996: 2.49 \% \text {, year } \\
1998: 2.48 \% \text {, year } 2000: 2.48 \% \\
\text { year } 2002: 5.16 \% \text { and year } \\
\text { 2004: } 4.73 \% \text { ), which are } \\
\text { considered acceptable. } \\
\text { However, one of the } \\
\text { comparison results ie CS2 at }\end{array}$} \\
\hline & $\mathrm{B}(\%)$ & 80.00 & 14.29 & 5.71 & 0.00 & 0.00 & & \\
\hline & $\mathrm{C}(\%)$ & 5.54 & 2.49 & 0.64 & 1.67 & 2.04 & & \\
\hline & $\mathrm{D}(\%)$ & & & 2.48 & & & & \\
\hline \multirow{4}{*}{2000} & $\mathrm{~A}(\%)$ & 74.46 & 16.78 & 5.07 & 1.67 & 2.04 & \multirow{4}{*}{$\begin{array}{c}100 \\
100 \\
- \\
-\end{array}$} & \\
\hline & $\mathrm{B}(\%)$ & 80.00 & 14.29 & 5.71 & 0.00 & 0.00 & & \\
\hline & $\mathrm{C}(\%)$ & 5.54 & 2.49 & 0.64 & 1.67 & 2.04 & & \\
\hline & $\mathrm{D}(\%)$ & & & 2.48 & & & & \\
\hline \multirow{4}{*}{2002} & $\mathrm{~A}(\%)$ & 73.12 & 13.13 & 5.82 & 4.67 & 3.28 & \multirow{4}{*}{$\begin{array}{l}100 \\
100 \\
- \\
-\end{array}$} & \multirow{4}{*}{$\begin{array}{l}\text { year } 2004 \text {, is slightly over } 10 \% \text {. } \\
\text { This could be the results of a } \\
\text { maintenance effect, which is } \\
\text { not considered in this proposed } \\
\text { deterioration model. Hence, the }\end{array}$} \\
\hline & $\mathrm{B}(\%)$ & 80.00 & 19.14 & 0.86 & 0.00 & 0.00 & & \\
\hline & $\mathrm{C}(\%)$ & 6.88 & 6.01 & 4.96 & 4.67 & 3.28 & & \\
\hline & $\mathrm{D}(\%)$ & & & 5.16 & & & & \\
\hline \multirow{4}{*}{2004} & $\mathrm{~A}(\%)$ & 81.10 & 7.32 & 2.99 & 2.95 & 5.65 & \multirow{4}{*}{$\begin{array}{l}100 \\
100 \\
- \\
-\end{array}$} & \multirow{4}{*}{$\begin{array}{l}\text { model is not able to predict this } \\
\text { change. Without taking into } \\
\text { consideration of this } \\
\text { maintenance effect, the } \\
\text { proposed feasibility study is } \\
\text { considered to produce } \\
\text { satisfactory results. }\end{array}$} \\
\hline & $\mathrm{B}(\%)$ & 80.00 & 19.14 & 0.86 & 0.00 & 0.00 & & \\
\hline & $\mathrm{C}(\%)$ & 1.10 & 11.80 & 2.13 & 2.95 & 5.65 & & \\
\hline & $\mathrm{D}(\%)$ & & & 4.73 & & & & \\
\hline
\end{tabular}

of long-term predictions from

\section{Discussion and Summary}

The main difficulty faced by current deterioration modelling techniques is the lack of usable data related to the bridge element's historical behaviour. Based heavily on a few sets of recent structural condition ratings, current modelling techniques cannot be expected to produce practically useful 
outcomes. This in turn leads to an unreliable prediction of future bridge condition ratings. In order to minimise this drawback, the BPM methodology has been developed to help improve the reliability of deterioration model for long-term prediction of bridge element performance. In order to confirm this, a feasibility study is presented in this paper for the BPM based long-term bridge element performance model using Time Delay Neural Networks (TDNNs).

The proposed TDNN provides only one-step ahead prediction at a time (one cycle). The result of the first one-step-ahead prediction (year 1996) is added on to the original TDNN input (years from 1968 to 1994). Iteration of the above-mentioned process is carried out until the end of the life cycle. The results of TDNN predictions (year from 1996 to 2024) are compared with the known BMS condition ratings (year from 1996 to 2004). The annual average prediction error is $3.47 \%$ which is less than $\pm 10 \%$, which is considered acceptable. Therefore, the forecasted condition ratings (19962024) by the TDNN can be considered as reliable prediction outcomes, particularly for elementlevel condition ratings. It should be noted, however, that this technique can only be used for "no maintenance' effects.

The present study conducted in this paper provides only the aim to establish the methodology of reliable long-term condition rating of bridge element using limited sample data. Further work should be carried out the case studies to confirm the methodology. However, the outcome of the present study would be useful for further development of a reliable bridge deterioration model for BMSs, which is highly dependent on the limited condition rating records.

\section{References}

[1] Das, P.C., Bridge Maintenance Management Objectives and Methodologies, in Bridge Management 3:inspection, maintenance, assessment and repair, J.E. Harding, G.A.R. Parke, and M.J. Ryall, Editors. 1996, E\&FN Spon: Lodon. p. 1-7.

[2] DeStefano, P.D. and D.A. Grivas, Method for estimating transition probability in bridge deterioration models. Journal of Infrastructure System, 1998. 4(2): p. 56-62.

[3] Hearn, G., et al. Bridge Maintenance and Management: A look to the future. in TRB 81st Annual Meeting: A3C06:Structures Maintenance and Management. 2000.

[4] Lee, J.H., et al., Improving the Reliability of a Bridge Management System (BMS) using an ANN-based Backward Prediction Model (BPM). Journal of Automation in Construction, 2008. 17(6): p. 758-772.

[5] Lounis, Z. and M.S. Mirza. Reliability-based service life prediction of deteriorating concrete structures. in Proceeding of 3rd International Conference on Concrete Under Severe Conditions. 2001. University of British Columbia, Vancouver. Canada.

[6] Sianipar, P.M.M. and T.M. Adams, Fault-tree model of bridge element deterioration due to interaction. Journal of Infrastructure System, 1997. 3(3): p. 103-110.

[7] Becerikli, Y. and Y. Oysal, Modeling and prediction with a class of time delay dynamic neural networks. Journal of Applied Soft Computing, 2007. 7: p. 1164-1169.

[8] Adams, T.M. and P.R. Sianipar, Tool for sensitivity analysis of bridge MR\&R policies, in Infrastructure condition assessment : art, science, and practice, M. Saito, Editor. 1997, ASCE: New York. p. 476-483. 